\title{
THE
}

UNIVERSITY

University of Rhode Island

OF RHODE ISLAND

DigitalCommons@URI

Physics Faculty Publications

Physics

$12-22-1975$

\section{Vortex Velocity in Turbulent He II Counterflow}

R. A. Ashton

J. A. Northby

University of Rhode Island, jnorthby@uri.edu

Follow this and additional works at: https://digitalcommons.uri.edu/phys_facpubs

Terms of Use

All rights reserved under copyright.

\section{Citation/Publisher Attribution}

Ashton, R. A., \& Northby, J. A. (1975). Vortex Velocity in Turbulent He II Counterflow. Physical Review

Letters, 35(25), 1714-1717. doi: 10.1103/PhysRevLett.35.1714

Available at: http://dx.doi.org/10.1103/PhysRevLett.35.1714

This Article is brought to you for free and open access by the Physics at DigitalCommons@URI. It has been accepted for inclusion in Physics Faculty Publications by an authorized administrator of DigitalCommons@URI. For more information, please contact digitalcommons-group@uri.edu. 
ence of the squares of the correlation lengths in two directions. With the appropriate $\xi_{n}$ and $\xi_{t}$ the Landau result is, of course, recovered.

A three-parameter fit of the corrected values of $R$ for MBBA by the function $C\left(T-T_{c}\right)^{-2 \nu}$ gives $\nu=0.57 \pm 0.09$ and $T_{p}-T_{c}=1.27 \pm 0.36^{\circ} \mathrm{C}$. Again the range of errors is the $95 \%$ confidence limit. This fit is shown in Fig. 3 as line $b$. The multiple-scattering correction does not affect the best value of $\nu$ and only increases $T_{c}$. Examination of Fig. 3 suggests that the departure of $\nu$ from 0.5 might be significant. However in view of the error bar it is difficult to ascertain a real departure from mean-field theory. ${ }^{11}$ The peculiar behavior in OBBA suggests further study of the coupling between nematic and smectic ordering in the isotropic phase. The coupling leads to a departure from the simple phenomenological theory already observed for the scattered intensity and the linewidth, ${ }^{12}$ but our results indicate that $\xi_{n}{ }^{2}-\xi_{t}{ }^{2}$ is for OBBA a much more sensitive quantity than the scattered intensity itself.

In conclusion, the first measurement of $L_{2} / a$ in the isotropic phase of a liquid crystal has been obtained. The sensitive method described here is expected to find applications in the study of liquid crystals, homologous series, and other depolarized scattering studies.

The participation of Aasmund Sudb $\varnothing$ in the early stages of the present work is gratefully acknowledged. Many thanks also go to Dr. M. Schadt for kindly supplying the OBBA sample, to Mr. H. Amrein for his technical assistance, and to Profes- sor J. Feder and Professor K. A. Müller for a critical reading of the manuscript.

\footnotetext{
*Present address: Physics Department, Technion, Haifa, Israel.

${ }^{1}$ T. W. Stinson and J. D. Litster, Phys. Rev. Lett. 25 , 503 (1970).

${ }^{2}$ T. W. Stinson and J. D. Litster, Phys. Rev. Lett. $\underline{30}$,
} 688 (1973).

${ }^{3}$ E. Gulari and B. Chu, J. Chem. Phys. 62,798 (1975).

${ }^{4}$ G. K. Wong and Y. R. Shen, Phys. Rev. A 10,1277 (1974).

${ }^{5}$ T. W. Stinson, J. D. Litster, and N. A. Clark, J. Phys. (Paris), Colloq. 33, C1-69 (1972).

${ }^{6}$ P. G. de Gennes, Mol. Cryst. Liq. Cryst. 12, 193 (1971).

7Its origin should be distinguished from the "Krishnan effect" [R. S. Krishnan, Proc. Indian Acad. A1, 211 (1934)], produced by Mie scattering from large molecules or aggregates, which is negligible here.

${ }^{8}$ J. B. Flannery, Jr., and W. Haas, J. Phys. Chem. 74, $3611(1970)$.

${ }^{9}$ Eastman Chemicals X11246, specially purified grade.

${ }^{10}$ L. D. Landau and E. M. Lifshitz, Electrodynamics of Continuous Media (Pergamon, Oxford, England, 1960), Chap. 14.

${ }^{11} \mathrm{~A}$ brief account of a theoretical study of critical behavior in the isotropic phase of nematics has been given by T. Lubensky and R. G. Priest, Phys. Lett. 48B, 103 (1974).

${ }^{12}$ T. R. Steger, Jr., J. D. Litster, and W. R. Young, in Liquid Crystals and Ordered Fluids, edited by J. F. Johnson and R. S. Porter (Plenum, New York, 1974), Vol. 2, p. 33 .

\title{
Vortex Velocity in Turbulent He II Counterflow*
}

\author{
R. A. Ashton and J. A. Northby \\ Department of Physics, University of Rhode Island, Kingston, Rhode Island 02881
}

(Received 8 October 1975)

\begin{abstract}
We have used the ion-vortex interaction to measure the drift velocity of the vorticity present in turbulent counterflow, and find that it moves in the direction of normal-fluid flow. The result is in direct conflict with a central assumption of the presently accepted model of the turbulent state.
\end{abstract}

Recent work on turbulence in He II counterflow has shown that the model first proposed by Vinen ${ }^{1}$ to account for second-sound attenuation is also able to give a reasonable description of critical velocities, ${ }^{2}$ ion trapping, ${ }^{3}$ and noise on secondsound signals. ${ }^{4}$ The principal assumptions of this model are (1) the superfluid state consists of an homogeneous, isotropic, tangled mass of vortex lines. (2) The vorticity is geometrically similar at all times and therefore may be characterized by $L_{0}$, the length of line per unit volume. (3) The state is maintained by a dynamic balance between growth and annihilation processes; dimensional arguments then serve to pre- 
dict their dependence on the heat current, $\dot{q}$.

(4) The vorticity moves on the average with the superfluid (toward the heater). This last assumption is needed in order to relate axial temperature gradients to transverse second-sound attenuation. Direct experimental evidence of vortex motion is lacking, but early studies ${ }^{5}$ of the deflection of negative-ion currents in turbulent counterflow did indicate a strong additional drag in the direction of the normal-fluid motion. This could mean that vortex motion is away from the heater.

We report here the first measurement of the sign and magnitude of the vortex velocity. In addition to resolving the conflict, we feel it provides a valuable clue to the detailed nature of the turbulent state. Our method, which also utilizes the ion-vortex interaction, indicates that in contrast to the model, vorticity drifts away from the heater. Furthermore, the magnitude of the velocity proves to be comparable to that of a vortex ring whose diameter is equal to the mean intervortex spacing.

Our experimental counterflow channel is shown schematically in Fig. 1. It is $10 \mathrm{~cm}$ long, with a $0.5 \times 1.0 \mathrm{~cm}^{2}$ rectangular cross section, and is open to the bath at the top. A wire heater resistor, $R$, initiates the counterflow. The electrodes are gold plated, and inlaid along the Lucite channel walls. A $200-\mathrm{mCi} 1 \times 0.6-\mathrm{cm}^{2}$ tritium $\beta$ source, $S$, is recessed in the wall behind a grid, $G$. The constant bias voltage, $V_{0}$, applied across the channel, permits a negative-ion current to flow

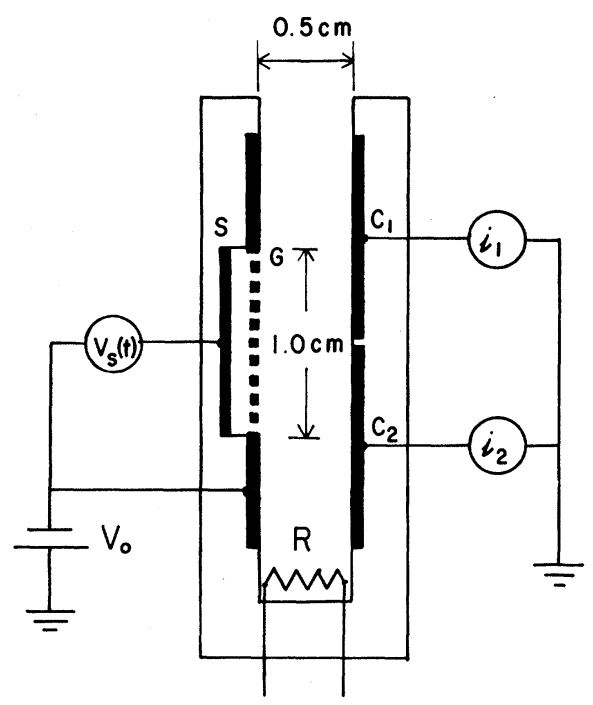

FIG. 1. Schematic of counterflow channel (not to scale). from $G$ to a pair of collectors, $C_{1}$ and $C_{2}$, placed symmetrically on the opposite wall. This current may then be switched on and off by modulating the voltage $V_{s}(t)$ applied to the source.

The interaction between the negative-ion beam and the vorticity present in turbulence has been discussed previously. ${ }^{3}$ Negative ions are trapped on vorticity by the same mechanism as in rotating helium, ${ }^{6}$ but in turbulence the escape process is not thermal excitation of the ion off the line. Instead it is related to the annihilation of the line segment on which the trapped charge resides. ${ }^{7}$ In the presence of constant ion and heat currents, competition between these two processes leads to the buildup of a uniform distribution of trapped charge in front of the grid. If the charging current is then switched off, after a transient comparable in length to the free-ion transit time, the total current reaching the two collectors will decay exponentially as the initially trapped charge is released through vortex annihilation. The decay time constant is a measure of the annihilation probability for an element of vorticity in the flow. If the vorticity is drifting with a velocity $v_{L}$, the plug of trapped charge must be drifting also. Since the fraction of the total current which reaches each individual collector will depend on the fraction of the remaining trapped charge in front of it, the downstream collector should receive an increasing fraction of the total current. We can obtain a quantitative measurement of $v_{L}$ by analyzing the time development of the differences in these currents in the following simplified model. We assume that the turbulence is homogeneous, and that the initial distribution of trapped charge is a uniform plug whose length, $L$, is equal to the length of the source. We also assume that the trapping centers drift toward $C_{1}$ with a uniform velocity, $v_{L}$. We presume the applied voltage $V_{0}$ to be large enough so that we may neglect retrapping of released charges, as well as their deflection by the normal fluid and other effects arising from a finite transit time. Finally we assume the release process is governed by an escape probability per unit time, $p_{e}$. In this model, currents arise from two processes; transverse motion of released charges, $i^{t}$, and axial motion of trapped charges, $i^{a}$. Consider first transverse motion. Each charge released in front of a collector will contribute an average charge $(-e / 2)$ to the current on that collector. Consequently

$$
i_{i}{ }^{t}=(-e / 2)\left(p_{e} N_{T} f_{i}\right),
$$


where $N_{T}(t)$ is the total number of trapped charges and $f_{i}(t)$ is the fraction in front of the $i$ th collector. Given our postulates we can write

$$
f_{i}(t)=(1 \pm \delta) / 2 \pm\left(v_{L} / L\right) t,
$$

where the positive sign refers to the downstream collector, $C_{1}$. The time-independent parameter $\delta$ accounts for any initial displacement of the plug. Next consider axial motion. For each trapped charged which crosses the boundary between the two collectors an average induced surface charge of $(+e / 2)$ will be transferred from $C_{2}$ to $C_{1}$. Since the rate at which charges cross the boundary is $\left(N_{T} v_{L} / L\right)$ we have

$$
i_{i}{ }^{a}=( \pm e / 2) N_{T} v_{L} / L,
$$

where the minus sign applies to $C_{1}$. Defining $\Delta i \equiv i_{1}-i_{2}$ and $i_{T} \equiv i_{1}+i_{2}$ we can combine these results into the convenient expression

$$
\left(\Delta i / i_{T}\right)=\left(\delta+2 v_{L} / p_{e} L\right)+\left(2 v_{L} / L\right) t .
$$

We therefore expect the difference in the currents divided by their sum, to be a linear function of time whose slope is a direct measure of $v_{L}$. This model is valid only until the charge plug drifts beyond $C_{2}$. After this time $t_{1} \equiv\left(L / 2 v_{L}\right), i_{2}=0$, and $\Delta i / i_{T}=1$. The result (4) can be extended to the case of a distribution of velocities by replacing $v_{L}$ and $p_{e}$ by their average values, provided that they are uncorrelated.

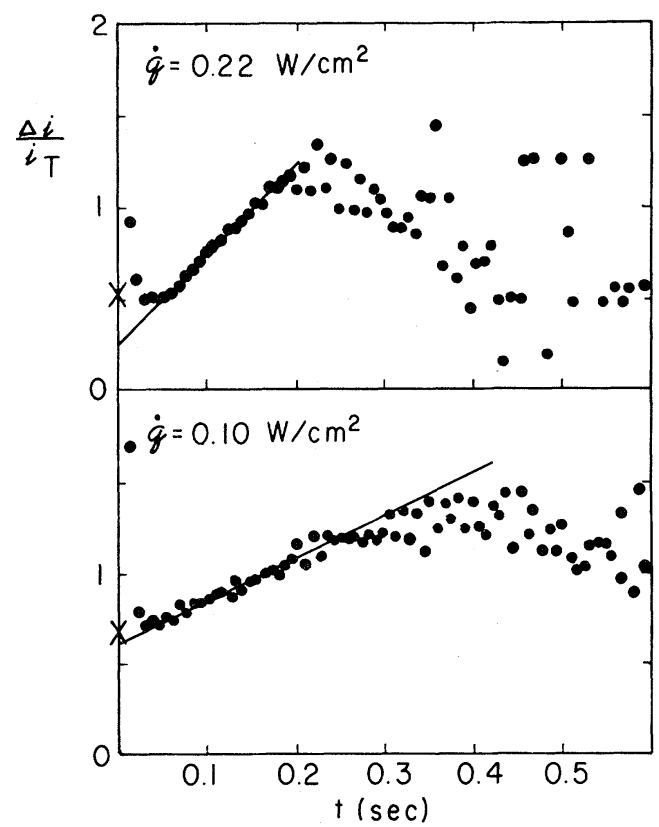

FIG. 2. Difference current divided by total current versus time at $T=1.31 \mathrm{~K}$, for two heat currents.
Figure 2 shows typical experimental plots of $\Delta i / i_{T}$ versus time at $T=1.31 \mathrm{~K}$, for two different heat currents. After an initial transient the curve is an increasing function of time, which is unambiguous evidence of motion of the trapped charges in the direction of normal-fluid flow. In fact, the linear behavior predicted in our simple model is clearly in evidence. The straight lines are least-squares fits to the region or apparent linearity. Their slopes give us $\left(2 v_{L} / L\right)$. They are terminated at the time $t_{1}$ calculated from this slope, since beyond that point the model requires $\Delta i / i_{T}=1$. It is encouraging that $t_{1}$ characterizes fairly well the end of linear behavior.

In Fig. 3 we show values of $v_{L}$ as a function of $\dot{q}$, for three different temperatures. The dependence on $\dot{q}$ is roughly linear and the magnitude ranges from about $\frac{1}{5}$ of the calculated normalfluid velocity at $1.31 \mathrm{~K}$, to about $\frac{1}{3}$ at $1.59 \mathrm{~K}$. We have also obtained data at three intermediate temperatures which are qualitatively similar, but which have been omitted for reasons of clarity. These data were taken with a transverse electric field of $280 \mathrm{~V} / \mathrm{cm}$. Reduction to 140 $\mathrm{V} / \mathrm{cm}$ produced no noticeable change in our results, however. Since retrapping depends on field strength, this insensitivity supports our assumption that it is negligible.

One conclusion which must follow from a nonzero value of $v_{L}-v_{s}$ is that the vortex array is almost certainly anisotropic. Since Vinen's argument relating axial temperature gradients to transverse second-sound attentuation assumes

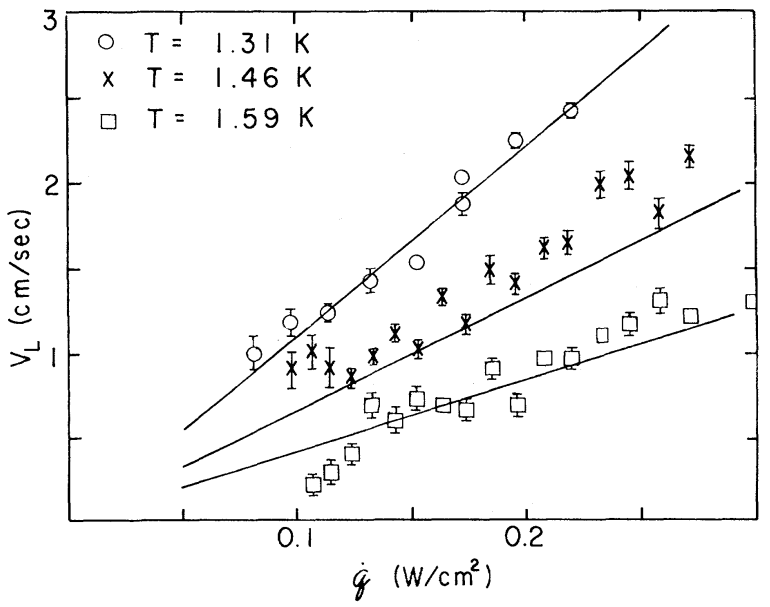

FIG. 3. Vortex velocity versus heat current at three different temperatures. Indicated errors represent \pm 1 standard deviation. 
isotropy as well as that $v_{L}=v_{s}$, that aspect of his model requires modification. Other than this, however, none of the postulates underlying his dimensional analysis are in direct conflict with these new results. We can, in fact, predict the dependence of $v_{L}$ on $\dot{q}$ and on $T$ by the following simple extension of that analysis. In the localized self-induction approximation ${ }^{8}$ valid for the motion of vortex filaments, the velocity of an element of vorticity relative to the superfluid is inversely proportional to the radius of curvature. An average drift velocity must then imply the existence of an average radius of curvature, ${ }^{9}$ $\bar{R}$. If we accept the hypothesis of geometrical similarity, the only relevant length in the problem is $L_{0}^{-1 / 2}$, and we must have $\bar{R} \propto L_{0}^{-1 / 2}$. Consequently, if we define $v_{L}$ as the average vortex velocity in the direction of normal-fluid flow measured in the lab frame, we have

$$
v_{L}=C L_{0}{ }^{1 / 2}-v_{s} \text {, }
$$

where $C$ is independent of both $\dot{q}$ and $T$, and $v_{s}$ is the superfluid velocity. The solid curves in Fig. 3 are calculated using Vinen's experimental values of $L_{0}$, with $C$ adjusted to equal $2.4 \kappa$, where $\kappa$ is the quantum of circulation. The agreement here is quite good, as it is for other intermediate temperatures. It must be emphasized that a single adjustable constant generates all of these curves - the dependence of $\left(v_{L}-v_{s}\right)$ on $\dot{q}$ and $T$ is entirely contained in $L_{0}{ }^{1 / 2}$.

These arguments do not allow us to predict the magnitude of the constant $C$. It is suggestive however that a plot of the velocity in the lab frame of a vortex ring whose diameter $D=(0.8) L_{0}^{-1 / 2}$ produces essentially the same curves shown in Fig. 3.

Expression (4) indicates that the intercept depends on $v_{L}$ also. Unfortunately, quantitative analysis is prevented by our lack of knowledge of $\delta$. This parameter accounts principally for initial axial displacement of the trapped-charge plug, which can result from several processes. In any case, however, we expect it to be small. The other term, $\left(2 v_{L} / p_{e} L\right)$, may be determined. experimentally, since $p_{e}^{-1}$ is just the decay constant for $i_{T}$. This quantity which is indicated by the crosses at $t=0$ in Fig. 2, should equal the experimental intercept if $\delta$ is indeed negligible. In general, agreement is good for small $\dot{q}$ but gets progressively poorer at larger $\dot{q}$, particularly at the highest temperatures. It is tempting to attribute the difference to a temperature and heat current dependent $\delta$, but we are presently unable to account for even the qualitative nature of these dependences. This difficulty may arise from a fundamental limitation of our model, but we feel it is more likely to be a manifestation of some second-order effect such as turbulence in the source region or channel-induced inhomogenieties. We are presently planning further experiments in different geometries which should help to clarify these points. In addition we expect to extend the method to study turbulence in pure-superfluid flow.

*Work supported by the National Science Foundation and by Research Corporation.

${ }^{1}$ W. F. Vinen, Proc. Roy. Soc., Ser. A 240, 114, 128 (1957), and 242, 493 (1957), and 243, 400 (1958).

${ }^{2}$ R. K. Childers and J. T. Tough, Phys. Rev. Lett。31, 911 (1973).

${ }^{3}$ R. A. Ashton and J. A. Northby, Phys. Rev. Lett. 30 , 1119 (1973).

${ }^{4}$ H. Hoch, L. Busse, and F. Moss, Phys. Rev. Lett. 34, 384 (1975).

${ }^{5}$ G. Careri, F. Scaramuzzi, and J. O. Thompson, Nuovo Cimento 18, 957 (1960).

${ }^{6}$ R. J. Donnelly, Experimental Superfluidity (Univ. of Chicago Press, Chicago, Ill., 1967), Chap. 6.

${ }^{7}$ D. M. Sitton and F. Moss, Phys. Rev. Lett. 29, 542 (1972).

${ }^{8}$ R. J. Arms and F. R. Hama, Phys. Fluids $\underline{8}, 553$ (1965); see also P. H. Roberts and R. J. Donnelly, in Annual Review of Fluid Mechanics, edited by M. Van Dyke and W. Vincenti (Annual Reviews, Inc., Palo Alto, Calif., 1974), Vol. 6.

${ }^{9}$ More precisely, if $\hat{n}$ is the unit vector normal to the line and $\vec{\kappa}$ the circulation, the average of $\vec{\kappa} \times \hat{n} / R)$ must be axial, with magnitude $\kappa /(\bar{R})$. 